\title{
The Penumbra System for the Treatment of Acute Ischemic Stroke: Report of Two Cases
}

\author{
Xudong ZHANG ${ }^{1}$, Jun Wei GAO², Pascal JABBOUR ${ }^{3}$ \\ ${ }^{1}$ Gansu Province People Hospital, Department of Neurology, Lanzhou, China \\ ${ }^{2}$ The Second Hospital of LanzhouUniversity, Department of Neurosurgery, Lanzhou, China \\ ${ }^{3}$ Thomas Jefferson University, Hospital For Neuroscience, Division of Neurovascular and Endovascular Surgery, Department of Neurosurgery, \\ Philadelphia, USA
}

\section{ABSTRACT}

Mortality associated with the occlusion of large vessels in acute ischemic stroke is particularly high despite best available medical therapy. Early and safe revascularization of the primary occlusion is correlated with good clinical result. We report two patients with acute ischemic stroke in whom the mechanical device Penumbra System was used for thrombolysis and embolectomy. The Penumbra System provided the revascularization of the primary occlusion site in the two patients and complete revascularization was obtained. Improvement was observed in both cases on the National Institutes of Health Stroke Scale and on modified Rankin scale scores at 1 and 30 days post-procedure. Neither of the patients had intracranial hemorrhage. The Penumbra System is a valuable device as a treatment for acute ischemic stroke secondary to large vessel occlusion.

KEYWORDS: Acute ischemic stroke, Occlusions of large vessels, Penumbra system, Revascularization

\section{INTRODUCTION}

Mortality associated with occlusions of large vessels in acute ischemic stroke is high despite the improvements in medical treatment $(2,3,6)$. Substantial evidence has shown that early and safe revascularization of the primary occlusion is correlated with a satisfactory clinical outcome $(1,5)$. The Penumbra System (PS; Penumbra, Alameda, California, USA) is a new embolectomy device which is designed to remove the thrombus in acute ischemic stroke secondary to large vessel obstruction (Figure 1) (1). We report two patients with acute ischemic stroke in whom the mechanical device Penumbra System was used for thrombolysis.

\section{CASE REPORTS}

Two patients were admitted to the Thomas Jefferson University Hospital for Neuroscience.

Case 1: A seventy-year-old female patient had a history of atrial fibrillation, and presented with sudden onset of right hemiplegia and aphasia. Baseline National Institutes of Health Stroke Scale (NIHSS) score was 14. Head computed tomography (CT) scan was normal. Digital subtraction angiography (DSA) showed left middle cerebral artery (MCA) superior M2 segment occlusion with intact lenticulostriate arteries. Times from onset to arterial puncture were 4 hours 10 minutes. After using the Penumbra system, normal flow was restored. NIHSS was 0 one day after procedure. The thirty-day modified Rankin scale (mRS) score was 0 (Figure 2A, B).

Case 2: A 65-year-old male presented with right hemiplegia. The baseline NIHSS score was 20 . No abnormality was found on CT scan. DSA demonstrated left MCA M1 segment occlusion. Arterial puncture was accessed in 5 hours when symptoms occurred. After using a mechanical device, complete revascularization was obtained. The NIHSS score was 5 one day post-procedure. The thirty day $\mathrm{mRS}$ score was 1 (Figure 3A, B). 


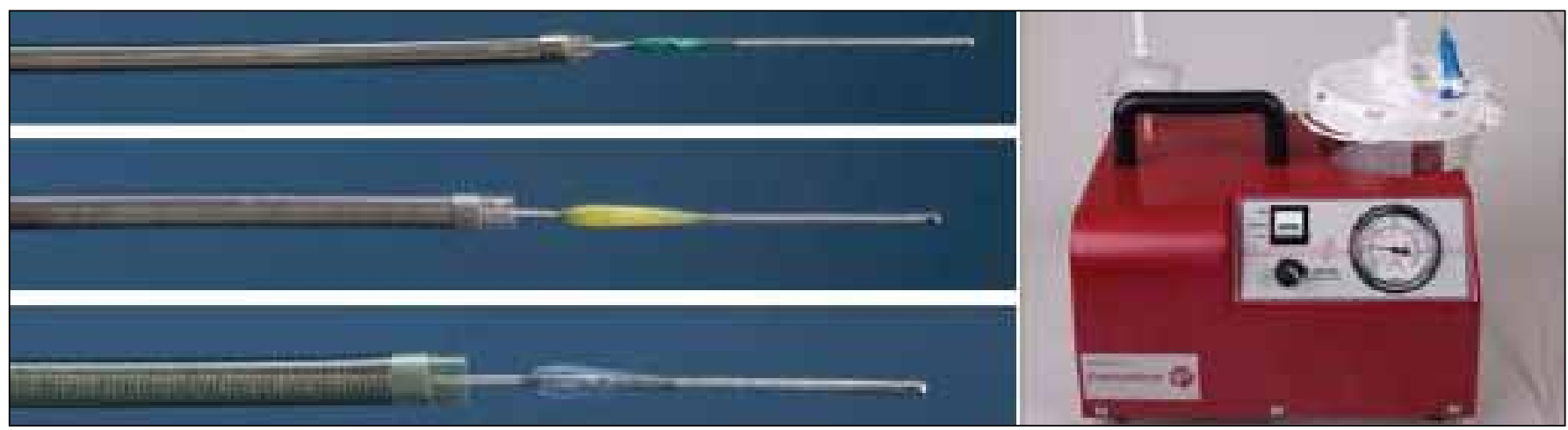

Figure 1: The Penumbra System (PS; Penumbra, Alameda, Calif).

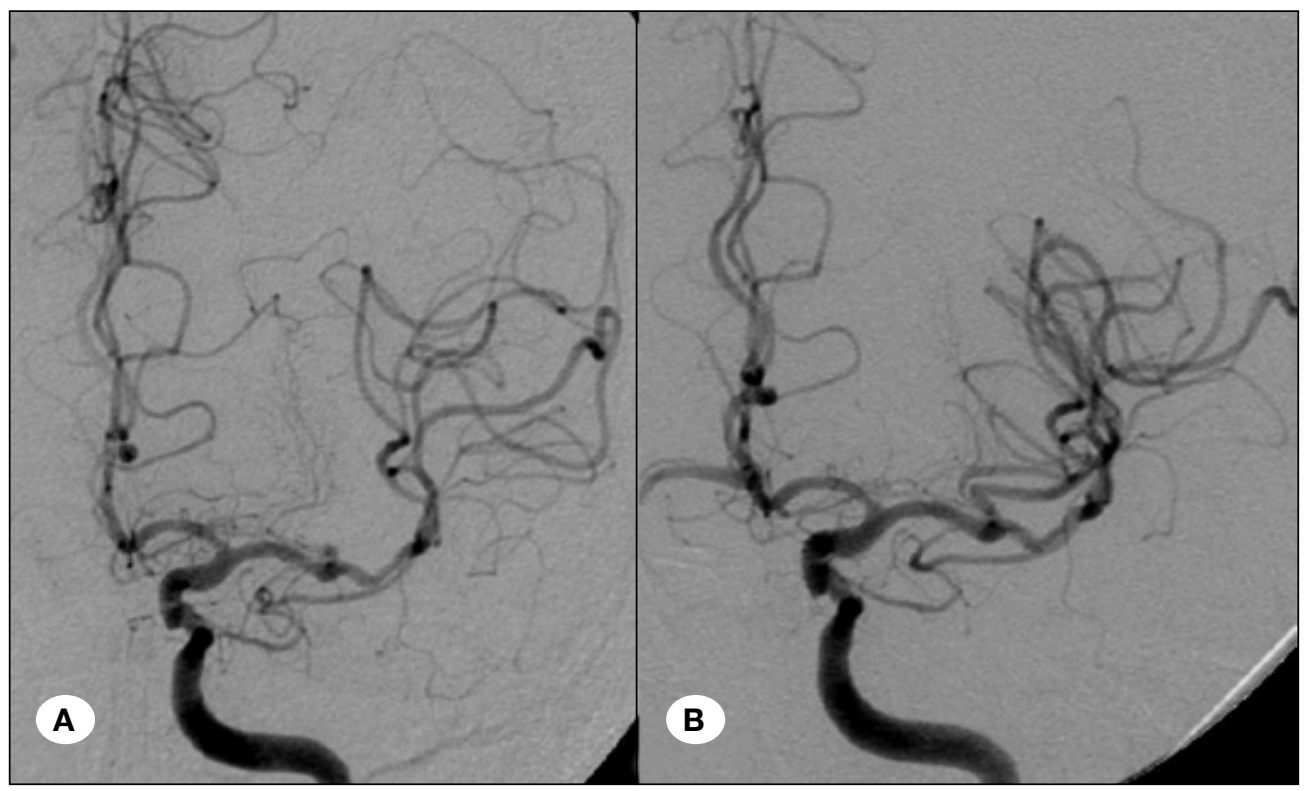

Figure 2: A) Before Penumbra left MCA superior M2 segment occlusion, B) post Penumbra normal flow was restored.

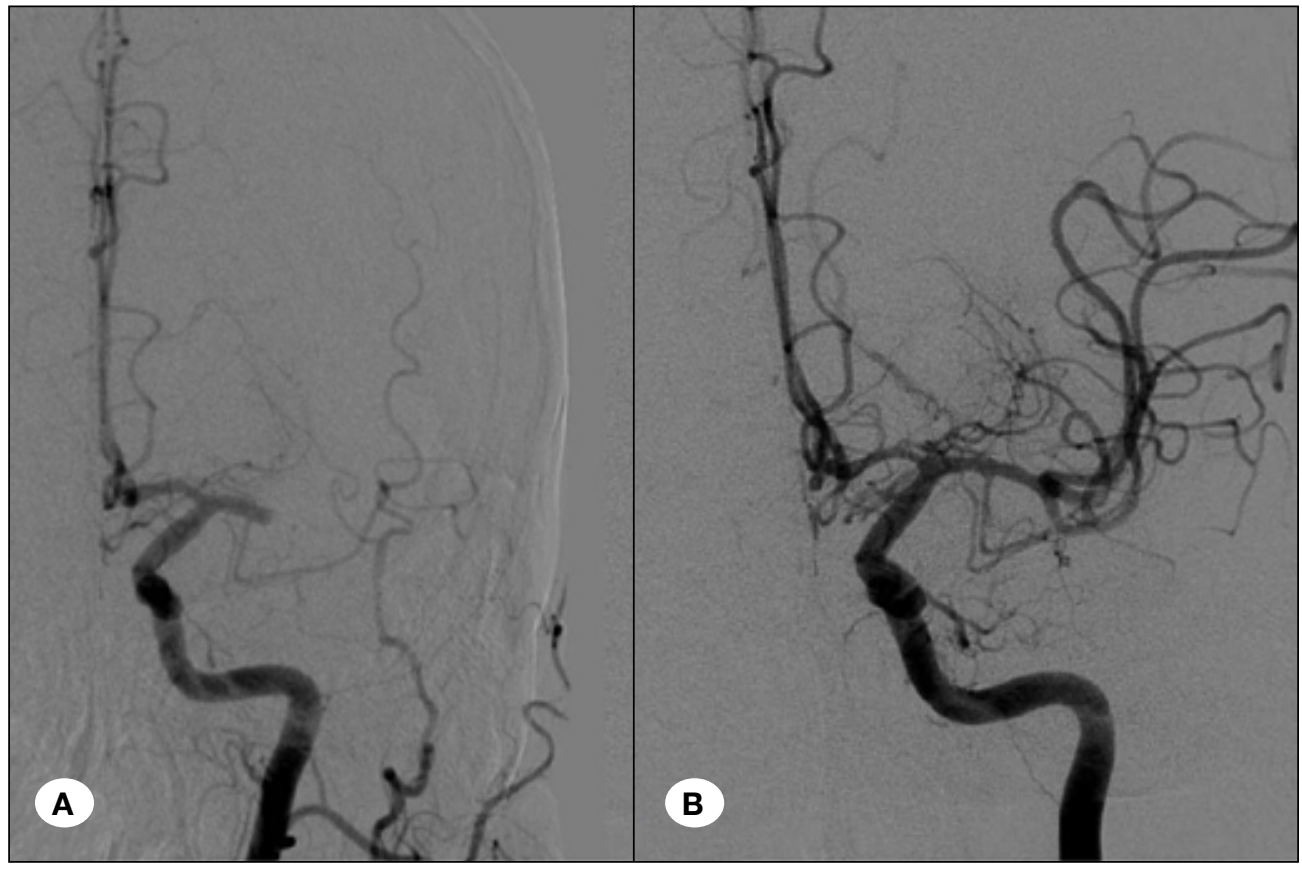

Figure 3: A) Before Penumbra left MCA M1 segment occlusion, B) post Penumbra successful revascularization. 
Treatment Procedures: Four-vessel DSA was used to determine the occluded vascular segment. Then, a guide catheter was brought into position in the occluded vessel territory to enable access by the reperfusion catheter. All of the components of the PS were deliverable via a $6 \mathrm{~F}$ guide catheter (1).

Once the appropriate position was achieved proximal to the clot, the guidewire was removed from the reperfusion catheter, and the separator was advanced through the reperfusion catheter. Then, the aspiration pump was turned on to initiate revascularization. Reduction of the clot burden by aspiration was accomplished by connecting the reperfusion catheter to the aspiration pump, which generated a vacuum of -20 inches $\mathrm{Hg}$. Continuous aspiration and debulking process became easy by advancing and withdrawing the separator through the reperfusion catheter into the proximal end of the clot (1).

Administration of anticoagulation and antiplatelet was suspended for 24 hours post-treatment.

\section{- DISCUSSION}

The National Institute of Neurological Disorders and Stroke study on the use of tissue plasminogen activator for acute ischemic stroke showed that the subjects experienced the greatest benefit from the treatment when the treatment with rtPA was initiated within 3 hours of stroke, $(1,7)$. However, patients in the treatment group did experience a higher rate of symptomatic intracranial hemorrhage $(\mathrm{ICH})(7)$.

Currently, studies have been shifted to mechanical rather than pharmaceutical means of recanalizing the site of primary occlusion in order to reduce adverse effects associated with the thrombolytic agents. Today, one mechanical device is available in the United States and Europe for revascularization of occlusions secondary to acute ischemic stroke $(1,4,9,10)$. Reports showed a revascularization rate of $48 \%$ with procedure-related adverse event rate of $7.1 \%$ (10). In the MERCl trial, 11 subjects experienced symptomatic $\mathrm{ICH}$, and $27.7 \%$ of them had asymptomatic $\mathrm{ICH}$. At 90 days after stroke, $43.5 \%$ of the subjects died, and $27.7 \%$ of the subjects had a good outcome $(1,9,10)$. In another trial, the rate of revascularization was increased to $69.0 \%$, and mortality was reduced to $30.6 \%$ at 3 months $(1,9)$. However, this was associated with an increase in symptomatic $\mathrm{ICH}$ to $9.0 \%$ and an increase in the asymptomatic ICH rate to $29.7 \%(1,9)$. By comparison, in our report, the PS was able to revascularize the site of primary occlusion in two patients, and both patients showed improvement on the NIHSS and an mRS scores at 1 and 30 days post-procedure (1). None of the two patients had intracranial hemorrhage. No procedure-related complications were noted. The Penumbra Pivotal Stroke Trial (8) shows that the Penumbra System provides safe and effective revascularization in patients experiencing ischemic stroke secondary to large vessel occlusive disease who present within 8 hours from symptom onset $(8,11)$.

In conclusion, the Penumbra system is a useful device for the treatment of acute ischemic stroke secondary to large-vessel occlusion, and further mass sample and randomized clinical trial is warranted.

\section{- REFERENCES}

1. Bose A, Henkes H, Alfke K, Reith W, Mayer TE, Berlis A, Branca V, Sit SP: Penumbra phase 1 stroke trial investigators: The penumbra system: A mechanical device for the treatment of acute stroke due to thromboembolism. AJNR Am J Neuroradiol 29: 1409-1413, 2008

2. Brandt $T$, von Kummer $R$, Muller-Kuppers $M$, Hacke $W$ : Thrombolytic therapy of acute basilar artery occlusion. Variables affecting recanalization and outcome. Stroke 27: 875-881, 1996

3. Eckert B, Koch C, Thomalla G, Kucinski T, Grzyska U, Roether J, Alfke $\mathrm{K}$, Jansen $\mathrm{O}$, Zeumer H: Aggressive therapy with intravenous abciximab and intra-arterial rtPA and additional PTA/stenting improves clinical outcome in acute vertebrobasilar occlusion: Combined local fibrinolysis and intravenous abciximab in acute vertebrobasilar stroke treatment (FAST): Results of a multicenter study. Stroke 36:1160-1165,2005

4. Gobin YP, Starkman S, Duckwiler GR, Grobelny T, Kidwell CS, Jahan R, Pile-Spellman J, Segal A, Vinuela F, Saver JL: MERCI 1: A phase 1 study of mechanical embolus removal in cerebral ischemia. Stroke 35:2848-2854, 2004

5. Jansen $O$, Schellinger $P$, Fiebach J: Early revascularization in acute ischemic stroke saves tissue at risk defined by MRI. Lancet 353:2036-2037, 1997

6. Jansen O, von Kummer R, Forsting M, Hacke W, Sartor K: Thrombolytic therapy in acute occlusion of the intracranial internal carotid artery bifurcation. AJNR Am J Neuroradiol 16:1977-1986, 1995

7. National Institute of Neurological Disorders and Stroke t-PA Stroke Study Group: Tissue plasminogen activator for acute ischemic stroke. N Engl J Med 333:1581-1587, 1995

8. Penumbra Pivotal Stroke Trial Investigators: The penumbra pivotal stroke trial safety and effectiveness of a new generation of mechanical devices for clot removal in intracranial large vessel occlusive disease. Stroke 40: 2761-2768, 2009

9. Smith WS: Safety of mechanical thrombectomy and intravenous tissue plasminogen activator in acute ischemic stroke. Results of the multi Mechanical Embolus Removal in Cerebral Ischemia (MERCl) trial, part I. AJNR Am J Neuroradiol 27: 1177-1182, 2006

10. Smith WS, Sung G, Starkman S, Saver JL, Kidwell CS, Gobin YP, Lutsep HL, Nesbit GM, Grobelny T, Rymer MM, Silverman IE, Higashida RT, Budzik RF, Marks MP; MERCI Trial Investigators: Safety and efficacy of mechanical embolectomy in acute ischemic stroke: results of the MERCI trial. Stroke 36:1432-1438, 2005

11. Zenteno M, Vinuela F: Neuroimaging. Chapter 33E. In: Daroff RB, Fenichel G, Jankovic J, Mazziotta JC (eds). Bradley's Neurology in Clinical Practice, $6^{\text {th }}$ ed. Philadelphia: Elsevier, 2012:556-559 